\title{
Dynamical transitions in a modulated Landau-Zener model with finite driving fields
}

\author{
Wei Li and Li-Xiang Cen* \\ Center of Theoretical Physics, College of Physical Science and Technology, Sichuan University, Chengdu 610065, China
}

\begin{abstract}
We investigate a special time-dependent quantum model which assumes the Landau-Zener driving form but with an overall modulation of the intensity of the pulsing field. We demonstrate that the dynamics of the system, including the two-level case as well as its multi-level extension, is exactly solvable analytically. Differing from the original Landau-Zener model, the nonadiabatic effect of the evolution in the present driving process does not destroy the desired population transfer. As the sweep protocol employs only the finite driving fields which tend to zero asymptotically, the cutoff error due to the truncation of the driving pulse to the finite time interval turns out to be negligibly small. Furthermore, we investigate the noise effect on the driving protocol due to the dissipation of the surrounding environment. The losses of the fidelity in the protocol caused by both the phase damping process and the random spin flip noise are estimated by solving numerically the corresponding master equations within the Markovian regime.
\end{abstract}

\section{INTRODUCTION}

Exactly solvable time-dependent quantum system attracts increasing interest owing to its role in the design for quantum control. In particular, to model dynamical processes or target quantum states for atomic and molecular systems [1, 2], nonadiabatic transitions induced by time-varying external fields are often involved and the theoretical proposal of the driving protocol with desired dynamics is generally a prerequisite to accomplish the corresponding quantum tasks 3 - 14.

Landau-Zener (LZ) model [3, 4] and its analogs, represented by the Hamiltonian below, are the most frequently exploited proposals in the driving protocol

$$
H(t)=\Omega_{x}(t) J_{x}+\Omega_{z}(t) J_{z} .
$$

Here $J_{x, z}$ denote the angular-momentum operators and $\Omega_{x, z}(t)$ account for two components of the driving field along the $x$ and $z$ axes, respectively. Owing to the explicit time dependency of $H(t)$, the general solution to this kind of systems is highly nontrivial even for the simplest two-level case, i.e., with the azimuthal quantum number $j=\frac{1}{2}$. For the standard LZ sweep with $\Omega_{x}$ being constant and $\Omega_{z}(t)$ varying linearly with time, the very two-level model is exactly solvable and the transition probability induced by the evolution over $t \in(-\infty, \infty)$ is known well as the LZ formula [3, 4]. Notably, the LZ model has a wide range of applications in physics as well as in chemistry, including the LZ interferometry [15 18], the transfer of charge [19], chemical reactions [20, 21], controllable manipulation of qubit and qutrit systems 22 25], and so on.

The so-called counter-diabatic protocol [5, 6] (also named as the transitionless protocol [7] or shortcuts to adiabaticity [8]) has been proposed to generate exact dynamical evolution which aims at the adiabatic eigenstates, e.g., of a given Hamiltonian of form (1). Typically,

*Electronic address: lixiangcen@scu.edu.cn this kind of protocols exploit a reverse-engineering strategy through introducing an auxiliary counter-diabatic driving term [e.g., an extra time-varying field along the $y$ axis which cancels out the nonadiabatic effect of $H(t)$ ] to ensure the desired evolution. We would also like to mention another reverse-engineering algorithm proposed in Ref. [9], where a parametric connection is established between the the evolution operator and the control field of the Hamiltonian. In comparison, while the latter method is able to generate the LZ-type protocol with two driving components formed of Eq. (11), its applications were restricted to the two-level systems $[9-11]$.

Except for the models constructed through the mentioned reverse-engineering methods, analytically exactly solvable time-dependent quantum systems are relatively rare and known examples are mostly concentrated on the two-level system, for example, the Rosen-Zener [26], Allen-Eberly [27], Demkov-Kunike [28], and BambiniBerman [29] models. In a recent work, a tangent-pulse driven model has been proposed [30] which is shown to be analytically solvable not only for the two-level case but also for the multi-level extension. The nonadiabatic dynamics generated by the model itself can serve as a desirable protocol for the population transfer without the need of any auxiliary fields. While the ideal design assumes an infinite chirping field, it is demonstrated that for an imperfect scanning process with truncation, the cutoff error caused to the population transfer could be suppressed to the infinity through enhancing the scanning rate of the protocol.

In this paper we propose a modulated LZ model and explore the generated dynamics for quantum control. In particular, we demonstrate that the model offers an alternative protocol for the nonadiabatic population transfer which retains the advantages previously displayed in the tangent-pulse driven model: the nonadiabatic evolution can realize complete population transfer and no auxiliary field is required; the model is genuinely solvable which can be extended to the multi-level system. Furthermore, since the present protocol employs only the fields of finite intensity, it avoids the nonrealistic design of infinite driving assumed in the original LZ model and 
other analogous schemes. Meanwhile, the cutoff error in the protocol due to the truncation of the scanning pulse to the finite time interval is shown to be negligibly small. To evaluate further the feasibility of the scheme in the real systems, we investigate the noise effect of the protocol under dissipation. We solve numerically the master equations associated with the dephasing process and the random spin flip process within the Markovian regime. The loss of the fidelity caused by the detrimental influence of the noise is estimated.

The rest of the paper is organized as follows. In Sec. II we will introduce the modulated LZ model and demonstrate that the dynamics of model governed by the timedependent Schrödinger equation is exactly solvable. We will employ the method proposed by Lewis and Riesenfeld (LR) [31, 32] and manifest explicitly the dynamical invariant of the model. In Sec. III we shall focus on the dynamical transition in the model and describe the corresponding process of nonadiabatic population transfer for the two-level case as well as for its multi-level extension. Especially, we show that the intermediate transitions induced by the nonadiabatic effect will not destroy the desired state transfer. The noise effect on the fidelity of the protocol due to the dissipation of the environment is investigated in Sec. IV. Finally, a summary of the paper is presented in Sec. V.

\section{DESCRIPTION OF THE MODEL AND ITS EXACT SOLUTION}

The driven model considered here is described explicitly by the Hamiltonian

$$
H(t)=\frac{\eta}{1+\nu^{2} t^{2}}\left(J_{x}+\kappa \nu t J_{z}\right)
$$

where the amplitude $\eta$ and the sweep frequency $\nu$ are fixed constants and the coefficient $\kappa$ relates to them via

$$
\kappa=\sqrt{1-(\nu / \eta)^{2}} .
$$

Here we have set $\hbar=1$ such that $\eta \equiv \eta / \hbar$ possesses the same dimension with $\nu$. As the model keeps the property of the original LZ model that the ratio between the field components along the $z$ and $x$ axes $\Omega_{z}(t) / \Omega_{x}(t)$ increases linearly with time, an overall modulation on the field amplitude is exploited in the present sweep process. The schematic of the scanning pulses $\Omega_{x, z}(t)$ over $t \in(-\infty, \infty)$ is depicted in Fig. 1. Note that the modulation here enables the model to avoid the nonrealistic ingredient of assuming an infinite driving field in the original model.

We now show that the dynamics of the system governed by the Schrödinger equation $i \partial_{t}|\psi(t)\rangle=H(t)|\psi(t)\rangle$ is exactly solvable. To this goal, we recall the dynamical invariant introduced by the LR method [31, 32]. That is, a time-dependent quantum system could be solved exactly if the system possesses a dynamical invariant, i.e., an observable $I(t)$ that satisfies

$$
i \frac{\partial I(t)}{\partial t}-[H(t), I(t)]=0 .
$$

The peculiar property of such an invariant is that its instantaneous eigenvector, denoted by $\left|\phi_{m}(t)\right\rangle$, differs from the basic solution to the Schrödinger equation only by a phase factor: $\left|\psi_{m}(t)\right\rangle=e^{i \Phi_{m}(t)}\left|\phi_{m}(t)\right\rangle$, in which $\Phi_{m}(t)$ is expressed as

$$
\Phi_{m}\left(t, t_{0}\right)=\int_{t_{0}}^{t}\left\langle\phi_{m}\left(t^{\prime}\right)\left|i \frac{\partial}{\partial t^{\prime}}-H\left(t^{\prime}\right)\right| \phi_{m}\left(t^{\prime}\right)\right\rangle d t^{\prime} .
$$

Exact analytical expression of the LR invariant has ever been found for the time-dependent quantum system of particular classes [33 35]. Intriguingly, the above system is shown to possess the following invariant

$$
\begin{aligned}
I(t) & =\vec{\alpha}(t) \cdot \vec{J} \\
& =\frac{1}{\sqrt{1+\nu^{2} t^{2}}}\left(\kappa J_{x}+\frac{\nu}{\eta} J_{y}+\nu t J_{z}\right) .
\end{aligned}
$$

It is direct to verify that the specified $\alpha_{i}(t)$ satisfy

$$
\begin{aligned}
& \dot{\alpha}_{x}(t)=-\Omega_{z}(t) \alpha_{y}(t), \\
& \dot{\alpha}_{y}(t)=\Omega_{z}(t) \alpha_{x}(t)-\Omega_{x}(t) \alpha_{z}(t), \\
& \dot{\alpha}_{z}(t)=\Omega_{x}(t) \alpha_{y}(t),
\end{aligned}
$$

thus the relation of Eq. (4) is fulfilled.

To calculate the LR phase presented in Eq. (5), one is led to notice that $|\vec{\alpha}(t)|=1$ and $I(t)$ of Eq. (6) can be written as $I(t)=-G(t) J_{z} G^{\dagger}(t)$, in which $G(t)=e^{i \varphi J_{z}} e^{i \theta(t) J_{y}}$ accounts for a canonical transformation with $\theta(t)=\arccos \frac{-\nu t}{\sqrt{1+\nu^{2} t^{2}}}$ and $\varphi=-\arcsin \frac{\nu}{\eta}$. The eigenvector of $I(t)$ is then obtained as $\left|\phi_{m}(t)\right\rangle=G(t)|m\rangle$,
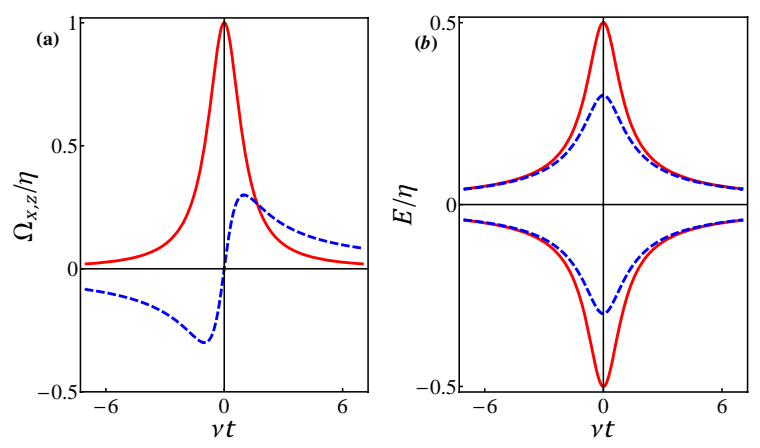

FIG. 1: The scanning process of the modulated Landau-Zener model specified by Eq. (1): (a) Time dependence of the two field components $\Omega_{x}(t) / \eta$ (solid line) and $\Omega_{z}(t) / \eta$ (dashed line) with $\kappa=0.6$ (i.e., $\nu / \eta=0.8$ ). (b) The corresponding adiabatic (solid line) and diabatic (dashed line) energy levels, $E_{ \pm}^{a d}(t)$ and $E_{ \pm}(t)$ over $\eta$ of the two-level system with $\kappa \rightarrow 1$ and $\kappa=0.6$, respectively. The levels exhibit maximal splits at $t=0$ with $E_{ \pm}^{a d}(0) / \eta=\mp 0.5$ and $E_{ \pm}(0) / \eta=\mp 0.3$. 
in which $|m\rangle(m=-j,-j+1, \cdots j)$ represents the eigenstate of $J_{z}$. With these notations, the two terms contained in the kernel of the integral of Eq. (5) can be worked out straightforwardly. It happens that the first term $\left\langle\phi_{m}(t)\left|i \partial_{t}\right| \phi_{m}(t)\right\rangle$, which denotes a nonadiabatic counterpart of the geometric connection of the adiabatic evolution, always vanishes in the present system. The second term $\left\langle\phi_{m}(t)|H(t)| \phi_{m}(t)\right\rangle$ identifies the diabatic energy levels 36] of the system and is shown to be

$$
E_{m}(t) \equiv\left\langle\phi_{m}(t)|H(t)| \phi_{m}(t)\right\rangle=\frac{-m \eta \kappa}{\sqrt{1+\nu^{2} t^{2}}} .
$$

As $\kappa \rightarrow 1$, they recover the adiabatic levels $E_{m}^{a d}(t)=$ $-m \eta / \sqrt{1+\nu^{2} t^{2}}$. We illustrate both $E_{m}^{a d}(t)$ and $E_{m}(t)$ for the $j=\frac{1}{2}$ case in Fig. 1(b).

The rigorous dynamical solution achieved above is applicable to the general angular-momentum system with an arbitrary azimuthal quantum number $j$. It indicates a significant difference from that of the original LZ model since the exact LZ formula of the latter model, which has been achieved as an asymptotical result of the Weber's parabolic cylinder functions [4], applies only to the twolevel system. Moreover, it is worthy to stress that the demonstration of the overall dynamical invariant for the above model is highly nontrivial as the original model does not possess such an invariant 37]. As will be shown in the below, it implies that the survival probability of the adiabatic state in this model, albeit the existence of intermediate transitions associated with nonadiabatic effects, tends asymptotically to the unit for the overall evolution. It suggests that the nonadiabatic evolution of the model can serve as a protocol for complete population transfer.

\section{DYNAMICAL TRANSITIONS IN THE TWO-LEVEL AND MULTI-LEVEL SYSTEMS}

\section{A. Protocols for nonadiabatic population transfer}

Following the expression of Eq. (6), $I(t)$ will evolve from $-J_{z}$ to $J_{z}$ along a geodesic curve in the Bloch space during the overall evolution $t \in(-\infty, \infty)$. Since the eigenstates of $I(t)$ are transported parallel without transitions, an initial eigenstate $|m\rangle$ then will evolve to the ending state $|-m\rangle$ at $t \rightarrow \infty$. Therefore, up to a phase term, the generated dynamics yields complete population transfer $|m\rangle \leftrightarrow|-m\rangle$ for the system whatever the sweep process is adiabatic or nonadiabatic.

For an irreducible space spanned by the angular momentum operator with a specific quantum number $j$, an explicit expression of the basis state $\left|\phi_{m}(t)\right\rangle$ could be obtained via

$$
\begin{aligned}
\left|\phi_{m}(t)\right\rangle & =e^{i \varphi J_{z}} e^{i \theta J_{y}}|m\rangle \\
& =\sum_{m^{\prime}} \mathcal{D}_{m^{\prime} m}^{j}(\theta) e^{i m^{\prime} \varphi}\left|m^{\prime}\right\rangle
\end{aligned}
$$

in which $\mathcal{D}_{m^{\prime} m}^{j}(\theta) \equiv\left\langle m^{\prime}\left|e^{i \theta J_{y}}\right| m\right\rangle$ has explicit expression for the specified $j$ [38] and the index $m^{\prime}$ of the summation is taken over $-j,-j+1, \cdots, j$. Specifically, for the twolevel system with $j=\frac{1}{2}$, one has

$$
\left|\phi_{ \pm}(t)\right\rangle=e^{ \pm i \frac{\varphi}{2}} \cos \frac{\theta(t)}{2}| \pm\rangle \pm e^{\mp i \frac{\varphi}{2}} \sin \frac{\theta(t)}{2}|\mp\rangle,
$$

where we have used the notation " $| \pm\rangle$ " for $\left| \pm \frac{1}{2}\right\rangle$. Accordingly, the phase-equipped dynamical basis $\left|\psi_{ \pm}(t)\right\rangle$ is obtained straightforwardly and the process of the population transfer is then characterized as

$$
\begin{aligned}
\left|\psi_{+}(-\infty)\right\rangle & =|+\rangle \rightarrow\left|\psi_{+}(\infty)\right\rangle=e^{i \beta_{+}}|-\rangle, \\
\left|\psi_{-}(-\infty)\right\rangle & =|-\rangle \rightarrow\left|\psi_{-}(\infty)\right\rangle=-e^{i \beta_{-}}|+\rangle
\end{aligned}
$$

in which $\beta_{ \pm}=\Phi_{ \pm}(\infty) \mp \varphi$ with

$$
\Phi_{ \pm}(\infty)= \pm \frac{1}{2} \eta \kappa \int_{-\infty}^{\infty}\left(1+\nu^{2} t^{2}\right)^{-\frac{1}{2}} d t
$$

The driving field in the present scheme has finite intensity and tends to zero asymptotically as $t \rightarrow \pm \infty$. In the practical scanning process the driving field should be pulsed in a finite time duration with truncation, that is, $t \in\left[-\tau_{c}, \tau_{c}\right]$. It turns out that the cutoff of the pulse results in very limited influence on the transition probability. For simplicity, let us take the above two-level case as an example. The transition probability induced by the sweep over the period $t \in\left[-\tau_{c}, \tau_{c}\right]$ is defined by $P \equiv\left|\left\langle-\left|U\left(\tau_{c},-\tau_{c}\right)\right|+\right\rangle\right|^{2}$ (or equally by $\left.\left|\left\langle+\left|U\left(\tau_{c},-\tau_{c}\right)\right|-\right\rangle\right|^{2}\right)$ in which $U\left(\tau_{c},-\tau_{c}\right)$ accounts for the generated evolution operator

$$
U\left(\tau_{c},-\tau_{c}\right)=\sum_{ \pm} e^{i \Phi_{ \pm}\left(\tau_{c},-\tau_{c}\right)}\left|\phi_{ \pm}\left(\tau_{c}\right)\right\rangle\left\langle\phi_{ \pm}\left(-\tau_{c}\right)\right| .
$$

A straightforward calculation yields that

$$
P=1-\left(1+\nu^{2} \tau_{c}^{2}\right)^{-1} \cos ^{2} \frac{\Phi_{+}\left(\tau_{c},-\tau_{c}\right)}{2} .
$$

Loss of the fidelity of the population transfer, defined by $P_{\delta} \equiv 1-P$ here, is limited by the factor $\left(1+\nu^{2} \tau_{c}^{2}\right)^{-1}$. It is seen that as $\nu \tau_{c} \geq 10 \pi$, the population transfer is realized with a high fidelity: $P_{\delta} \lesssim 10^{-3}$.

\section{B. Nonadiabaticity-induced transitions and survival probabilities of the adiabatic states in the multi-level systems}

Dynamic control of the multi-channel nonadiabatic process is usually a more challenging task and there have been extensive studies [39 41] on that of the multi-state version of the Landau-Zener model. Intriguingly, the model we proposed above applies directly to the multilevel system and the multi-channel dynamical transitions can be manifested by exploring the model with high quantum number $j$. For the cases of $j=1$ and $j=\frac{3}{2}$, 

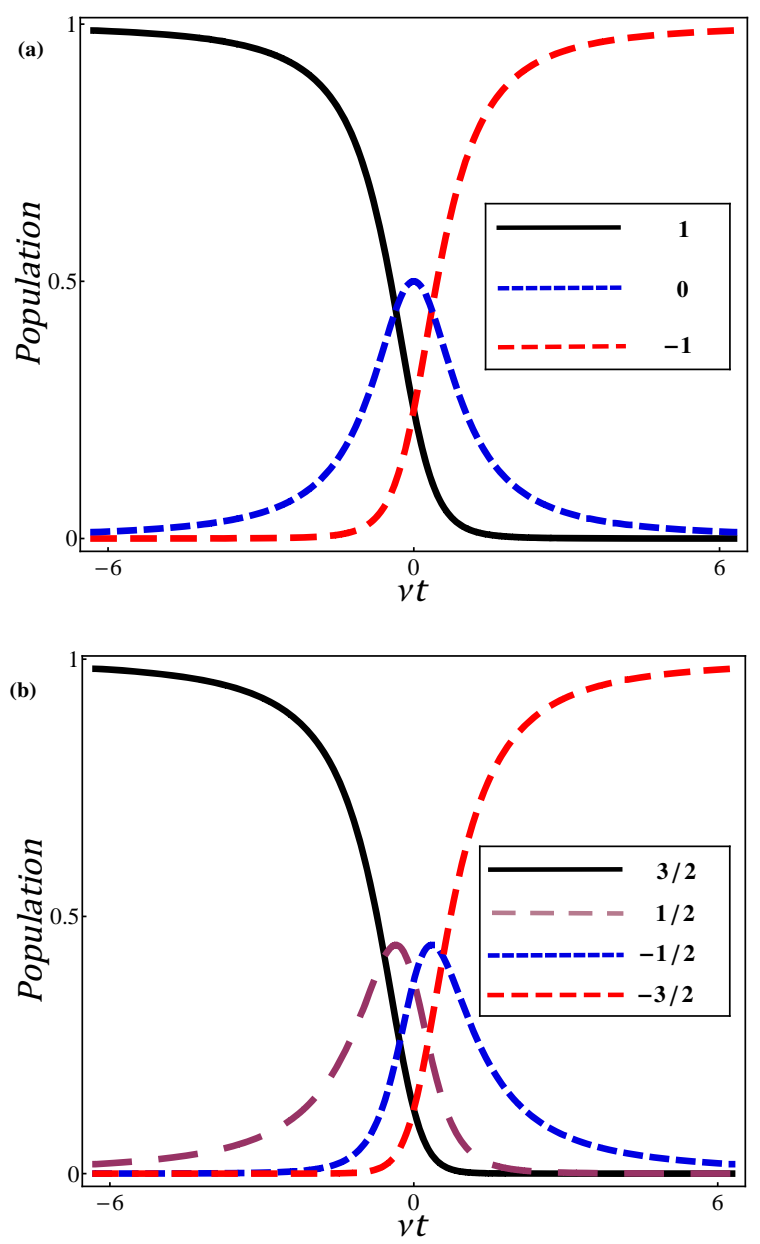

FIG. 2: Nonadiabatic population transfer along the time evolution in the multi-level systems in which the parameters are set as $\nu / \eta=0.8$ and $\kappa=0.6$. (a) The three-level system with $j=1$ and the initial state is in $|1\rangle$. The maximal population in the intermediate state $|0\rangle$ is obtained as $p=\frac{1}{2}$ at $t=0$. (b) The four-level system with $j=\frac{3}{2}$ and the initial state is in $\left|\frac{3}{2}\right\rangle$. The maximal populations on the states $\left|\frac{1}{2}\right\rangle$ and $\left|-\frac{1}{2}\right\rangle$ are obtained as $p=\frac{4}{9}$ at $\nu t=-\frac{1}{2 \sqrt{2}}$ and $\frac{1}{2 \sqrt{2}}$, respectively.

the corresponding representative matrices $\mathcal{D}^{j}(\theta)$ are expressed explicitly as

$$
\mathcal{D}^{1}(\theta)=\left(\begin{array}{ccc}
\cos ^{2} \frac{\theta}{2} & \frac{\sin \theta}{\sqrt{2}} & \sin ^{2} \frac{\theta}{2} \\
-\frac{\sin \theta}{\sqrt{2}} & \cos \theta & \frac{\sin \theta}{\sqrt{2}} \\
\sin ^{2} \frac{\theta}{2} & -\frac{\sin \theta}{\sqrt{2}} & \cos ^{2} \frac{\theta}{2}
\end{array}\right)
$$

and

$$
\mathcal{D}^{\frac{3}{2}}(\theta)=\left(\begin{array}{llll}
\cos ^{3} \frac{\theta}{2} & d_{12} & d_{13} & \sin ^{3} \frac{\theta}{2} \\
-d_{12} & d_{22} & d_{23} & d_{13} \\
d_{13} & -d_{23} & d_{22} & d_{12} \\
-\sin ^{3} \frac{\theta}{2} & d_{13} & -d_{12} & \cos ^{3} \frac{\theta}{2}
\end{array}\right)
$$

where $d_{12}=\frac{\sqrt{3}}{2} \cos \frac{\theta}{2} \sin \theta, d_{13}=\frac{\sqrt{3}}{2} \sin \frac{\theta}{2} \sin \theta, d_{22}=$ $3 \cos ^{3} \frac{\theta}{2}-2 \cos \frac{\theta}{2}$ and $d_{23}=2 \sin \frac{\theta}{2}-3 \sin ^{3} \frac{\theta}{2}$. The processes of the population transfer in these two cases are
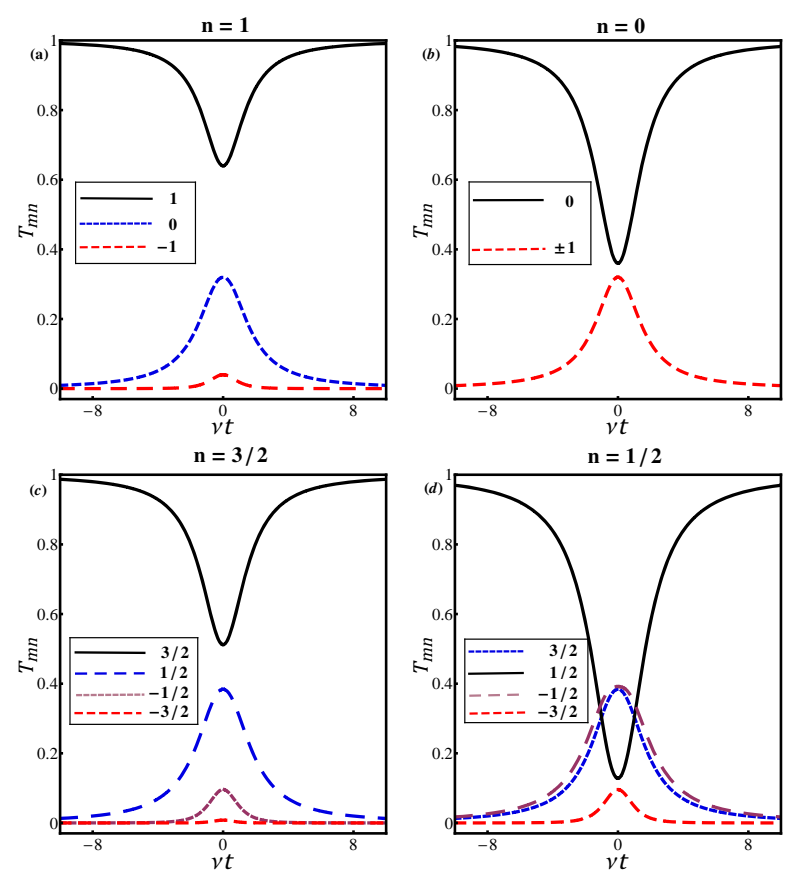

FIG. 3: Survival probability of the adiabatic states (the diagonal elements $T_{n n}$ ) and the nonadiabaticity-induced transition (the off-diagonal elements $T_{m n}$ with $m \neq n$ ) in the dynamical evolution. The parameters are set as $\nu / \eta=0.8$ and $\kappa=0.6$. (a) The $j=1$ model with an initial state $|1\rangle(n=1)$. (b) The $j=1$ model with an initial state $|0\rangle$ in which the intermediate transitions to $\left|\psi_{1}^{a d}(t)\right\rangle$ and to $\left|\psi_{-1}^{a d}(t)\right\rangle$ have an equal probability. (c) The $j=\frac{3}{2}$ model in which the initial state is in $\left|\frac{3}{2}\right\rangle\left(n=\frac{3}{2}\right)$ and all the elements $T_{m n}\left(m= \pm \frac{3}{2}, \pm \frac{1}{2}\right)$ are characterized. (d) The $j=\frac{3}{2}$ model in which the initial is in $\left|\frac{1}{2}\right\rangle\left(n=\frac{1}{2}\right)$.

depicted in Fig. 2, in which the initial states are taken to be $|1\rangle$ and $\left|\frac{3}{2}\right\rangle$, respectively.

To characterize further the nonadiabatic effects in the dynamical evolution, we evaluate the matrix of the transition probability: $T_{m n}=\left|\left\langle\psi_{m}^{a d}(t) \mid \psi_{n}(t)\right\rangle\right|^{2}$, in which $\left|\psi_{m}^{a d}(t)\right\rangle$ stands for the instantaneous adiabatic eigenvector of the Hamiltonian (2). The diagonal elements of the matrix $T$ represent the survival probabilities of the adiabatic basis states and the off-diagonal ones describe unambiguously the nonadiabaticity-induced transitions between these adiabatic states along the evolution. It is recognized that the basis set $\left|\psi_{m}(t)\right\rangle$, that are identical to $\left|\psi_{m}^{a d}(t)\right\rangle$ at the initial time $t \rightarrow-\infty$, will exhibit intermediate transitions during the evolution. However, as $\left|\psi_{m}(t)\right\rangle$ will recover $\left|\psi_{m}^{a d}(t)\right\rangle$ (up to a phase factor) eventually at $t \rightarrow \infty$, the desired population transfer is not destroyed by these nonadiabaticity-induced transitions. In Fig. 3 we illustrate in detail these phenomena for the model with $j=1$ and $j=\frac{3}{2}$. 

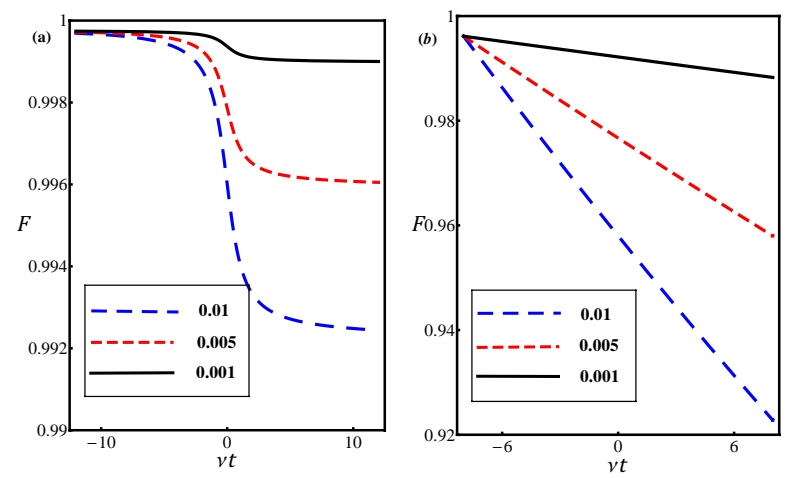

FIG. 4: Time evolving of the fidelity $F(t)$ of the driving protocol $(\kappa=0.6$ and $\nu / \eta=0.8)$ in the presence of noise effects. (a) Pure phase damping process in which $\gamma_{x, y}=0$ and $\gamma_{z} / \nu=0.01,0.005$ and 0.001 , respectively. The duration of the pulse is chosen to be $\nu \tau_{c}=10 \pi$ and the final fidelity is achieved as $F\left(\tau_{c}\right) \approx 0.992,0.996$ and 0.999 , respectively. (b) The random spin flip process with $\gamma_{x, y, z}=\gamma$. The duration of the pulse is chosen to be $\nu \tau_{c}=8$ and the fidelities $F\left(\tau_{c}\right) \approx 0.923,0.958$ and 0.988 are obtained for $\gamma / \nu=0.01,0.005$ and 0.001 , respectively.

\section{NOISE EFFECTS IN THE PRESENCE OF DISSIPATION}

In realistic systems the noise due to the surrounding environment is inevitable. The influence of the systembath coupling to the transition probability for the original LZ model has ever been studied in various background $[42[45]$. In the following we shall investigate the noise effect on the dynamics for the present modulated LZ model. Typically, we focus on the two-level system and estimate the population transfer in the presence of the spin flip noise which can arise as the interaction of the spin system with its fermionic reservoir is involved [46, 47]. Within the Markovian regime, the evolution of the system is described by the master equation

$$
\frac{\partial \rho(t)}{\partial t}=-i[H(t), \rho(t)]-\sum_{i} \frac{\gamma_{i}}{2}\left[J_{i},\left[J_{i}, \rho(t)\right]\right],
$$

where $\gamma_{i}(i=x, y, z)$ accounts for the damping rate of the corresponding spin flip process. Since we only consider the $j=\frac{1}{2}$ case, it is convenient to introduce the Bloch vector $\left(\rho_{x}, \rho_{y}, \rho_{z}\right)$ to describe the elements of the density operator, that is, $\rho_{x}=\rho_{+-}+\rho_{-+}, \rho_{y}=-i\left(\rho_{+-}-\rho_{-+}\right)$ and $\rho_{z}=\rho_{++}-\rho_{--}$. According to Eq. (17), one obtains that these components satisfy

$$
\frac{\partial}{\partial t}\left(\begin{array}{c}
\rho_{x} \\
\rho_{y} \\
\rho_{z}
\end{array}\right)=-\left(\begin{array}{lll}
\frac{\gamma_{y}+\gamma_{z}}{2} & \Omega_{z} & 0 \\
-\Omega_{z} & \frac{\gamma_{x}+\gamma_{z}}{2} & \Omega_{x} \\
0 & -\Omega_{x} & \frac{\gamma_{x}+\gamma_{y}}{2}
\end{array}\right)\left(\begin{array}{c}
\rho_{x} \\
\rho_{y} \\
\rho_{z}
\end{array}\right) .
$$

For the situation $\gamma_{x}=\gamma_{y}=0$, the above loss mechanism accounts for the pure phase damping process which does not lead to direct transitions between the two levels
$| \pm\rangle$. Nevertheless, as the dephasing process will alter the trajectory of the dynamical evolution generated by $H(t)$, it will result in imperfect effect on the desired population transfer. To characterize the influence of the noise on the dynamical evolution, the central task is to compute the fidelity $F(t)=\left|\left\langle\phi_{+}(t)|\rho(t)| \phi_{+}(t)\right\rangle\right|$, in which $\left|\phi_{+}(t)\right\rangle$ is given explicitly in Eq. (10). It is recognized that $F(t)$ describes the overlap between the actual time-evolving state and the target dynamical basis $\left|\phi_{+}(t)\right\rangle$. Starting from an initial state $|+\rangle$, we solve numerically the set of equations (18) for both the dephasing process and the random spin flip process with $\gamma_{x, y, z}=\gamma$. For the dephasing process, it happens that reduction of the fidelity mainly occurs in the vicinity of the point $t=0$. We have chosen the time duration $\nu \tau_{c}=10 \pi$ and the influence of the cutoff error is negligible. The result shows that the driving protocol is insensitive to the dephasing and a fidelity higher than 0.99 is obtained even the ratio $\gamma / \nu \gtrsim 10^{-2}$. On the other hand, the spin flip noise with the homogeneous damping rate will exert detrimental effects on the desired state transfer continuously over the whole time evolution. In our calculation we choose $\nu \tau_{c}=8$. Besides the noise effect, the dramatic truncation of the scanning process here has slight influence on the population transfer. The yielded results about the time evolving of the fidelity $F(t)$ are illustrated in Fig. 4 , in which different values of the ratio $\gamma / \nu$ are assumed.

\section{CONCLUSION}

We have investigated the exact dynamics of a modulated LZ model and exploited it as a design for nonadiabatic quantum control. Differing from the original LZ model, we have shown that this modulated model possesses an analytical dynamical invariant over the whole time domain and the generated dynamics is fully solvable analytically. While serving as a protocol for population transfer, the model is shown to possess the following distinct advantages: 1) nonadiabatic dynamics generated by the model itself can realize complete population transfer; 2) the protocol uses only finite driving fields which avoids the nonrealistic ingredient assuming infinite driving in the original LZ and also other analogous protocols. Furthermore, the scheme is applicable to the multi-level systems which offers an unambiguous scenario to manifest the multi-channel transitions induced by the nonadiabatic effects in the state transfer process.

As the noise due to the dissipative environment will lead to detrimental effects on the desired control process, we have also investigated the loss of the fidelity for the protocol when the system is subjected to the dissipation. The numerical calculations reveal that the protocol is not sensitive to the pure phase damping noise. On the other hand, to obtain high-fidelity population transfer in the presence of the spin flip noise with a homogeneous damping rate $\gamma$, our calculation shows that a requirement of the scanning rate of the protocol, $\nu / \gamma \gtrsim 10^{3}$, 
should be satisfied in general. Suppose that the coherence time $\gamma^{-1}$ is of an order $\sim 10^{2} \mu \mathrm{s}$ (which is achievable for the electron spin of the nitrogen-vacancy center in diamond 12]), then approximative evaluation yields that the sweep frequency should be $\nu \gtrsim 10 \mathrm{MHz}$ and the time duration of the pulse $\tau_{c} \sim \pi \mu \mathrm{s}$. By taking $\kappa=0.6$ (cf. Fig. 1) one gets $\eta \sim 12.5 \mathrm{MHz}$. Potential experimental implementation of the protocol in physical systems is highly expected.
[1] H. Nakamura, Nonadiabatic Transition (World Scientific, Singapore, 2002).

[2] P. W. Brumer and M. Shapiro, Principles of the Quantum Control of Molecular Processes (Wiley-Interscience, New York, 2003).

[3] L.D. Landau, Phys. Z. Sowjetunion 2, 46 (1932).

[4] C. Zener, Proc. R. Soc. A 137, 696 (1932).

[5] M. Demirplak and S. A. Rice, J. Phys. Chem. A 107, 9937 (2003).

[6] M. Demirplak and S. A. Rice, J. Phys. Chem. B 109, 6838 (2005).

[7] M. Berry, J. Phys. A: Math. Theor. 42, 365303 (2009).

[8] X. Chen, I. Lizuain, A. Ruschhaupt, D. Guery-Odelin, and J.G. Muga, Phys. Rev. Lett. 105, 123003 (2010).

[9] E. Barnes and S. Das Sarma, Phys. Rev. Lett. 109, 060401 (2012).

[10] E. Barnes, Phys. Rev. A 88, 013818 (2013).

[11] A. Messina and H. Nakazato, J. Phys. A 47, 445302 (2014).

[12] J. Zhang, J.H. Shim, I. Niemeyer, T. Taniguchi, T. Teraji, H. Abe, S. Onoda, T. Yamamoto, T. Ohshima, J. Isoya, and D. Suter, Phys. Rev. Lett. 110, 240501 (2013).

[13] M.G. Bason, M. Viteau, N. Malossi, P. Huillery, E. Arimondo, D. Ciampini, R. Fazio, V. Giovannetti, R. Mannella, and O. Morsch, Nat. Phys. 8, 147 (2012).

[14] B.B. Zhou, A. Baksic, H. Ribeiro, et al., Nat. Phys. 13, 330 (2017).

[15] W.D. Oliver, Y. Yu, J.C. Lee, K.K. Berggren, L.S. Levitov, and T.P. Orlando, Science 310, 1653 (2005).

[16] S.N. Shevchenko, S. Ashhab, and F. Nori, Phys. Rep. 492, 1 (2010).

[17] S. Gasparinetti, P. Solinas, and J.P. Pekola, Phys. Rev. Lett. 107, 207002 (2011).

[18] F. Forster, G. Petersen, S. Manus, P. Hänggi, D. Schuh, W. Wegscheider, S. Kohler, and S. Ludwig, Phys. Rev. Lett. 112, 116803 (2014).

[19] A.M. Kuztetsov, Charge Transfer in Physics, Chemistry, and Biology (Gordon and Breach, Reading, 1995).

[20] C. Zhu and S.H. Lin, J. Chem. Phys. 107, 2859 (1997).

[21] A. Nitzan, Chemical Dynamics in Condensed Phases (Oxford University Press, Oxford, 2006).

[22] L.F. Wei, J.R. Johansson, L.X. Cen, S. Ashhab, and Franco Nori, Phys. Rev. Lett. 100, 113601 (2008).

[23] G. Sun, X. Wen, B. Mao, J. Chen, Y. Yu, P. Wu, and S.
Han, Nat. Commun. 1, 51 (2010).

[24] J.Q. You and F. Nori, Nature (London) 474, 589 (2011).

[25] M.B. Kenmoe, L.C. Fai, Phys. Rev. B 94, 125101 (2016)

[26] N. Rosen and C. Zener, Phys. Rev. 40, 502 (1932).

[27] L. Allen and J. H. Eberly, Optical Resonance and TwoLevel Atoms (Dover, New York, 1975).

[28] Y.N. Demkov and M. Kunike, Vestn. Leningr. Univ., Ser. 4, Fiz. Khim. 16, 39 (1969).

[29] A. Bambini and P. R. Berman, Phys. Rev. A 23, 2496 (1981).

[30] G. Yang, W. Li, and L.-X. Cen, arXiv: 1608.00735.

[31] H.R. Lewis Jr., Phys. Rev. Lett. 18, 510 (1967).

[32] H.R. Lewis Jr. and W.B. Riesenfeld, J. Math. Phys. 10, 1458 (1969).

[33] S.J. Wang, Phys. Rev. A 42, 5107 (1990).

[34] L.-X. Cen, X.Q. Li, Y.J. Yan, H.Z. Zheng, and S.J. Wang, Phys. Rev. Lett. 90, 147902 (2003).

[35] T.T. Nguyen-Dang, E. Sinelnikov, A. Keller, and O. Atabek, Phys. Rev. A 76, 052118 (2007).

[36] The term "diabatic" has different usage in some other literatures, e.g., in the community of the multi-level Laudau-Zener model (see Refs. 39-41]).

[37] X.Q. Wang and L.-X. Cen, Phys. Lett. A 375, 2220 (2011).

[38] E.P. Winger, Group Theory and Its Application to the Quantum Mechanics of Atomic Spectra (Academic Press, New York, 1959).

[39] Y.N. Demkov and V.N. Ostrovsky, Phys. Rev. A 61, 032705 (2000).

[40] N.A. Sinitsyn, J. Phys. A 48, 195305 (2015).

[41] N.A. Sinitsyn, J. Lin, V.Y. Chernyak, Phys. Rev. A 95, 012140 (2017).

[42] M. Wubs, K. Saito, S. Kohler, P. Hänggi, and Y. Kayanuma, Phys. Rev. Lett. 97, 200404 (2006).

[43] S. Ashhab, Phys. Rev. A 90, 062120 (2014).

[44] S. Javanbakht, P. Nalbach, and M. Thorwart, Phys. Rev. A 91, 052103 (2015).

[45] Z. Sun, L. Zhou, G. Xiao, D. Poletti, and J. Gong, Phys. Rev. A 93, 012121 (2016).

[46] N. Yamada, A. Sakuma, and H. Tsuchiura, J. Appl. Phys. 101, 09C110 (2007).

[47] M.W. Wu, J.H. Jiang, and M.Q. Weng, Phys. Rep. 493, 61 (2010). 